Accepted March 6, 2002 for publication in Ap.J.Letters

\title{
Discovery of four X-ray quasars behind the Large Magellanic Cloud ${ }^{1}$
}

\author{
A. Dobrzycki ${ }^{2}$, P. J. Groot ${ }^{2,3}$, L. M. Macri ${ }^{2}$ and K. Z. Stanek ${ }^{2}$
}

\begin{abstract}
We present the discovery of four X-ray quasars $\left(z_{\mathrm{em}}=0.26,0.53,0.61,1.63\right)$ located behind the Large Magellanic Cloud; three of them are located behind the bar of the LMC. The quasars were identified via spectroscopy of optical counterparts to X-ray sources found serendipitously by the Chandra X-ray Observatory satellite. All four quasars have archival VI photometry from the OGLE-II project; one of them was found by OGLE to be variable. We present the properties of the quasars and discuss their possible applications.
\end{abstract}

Subject headings: Magellanic Clouds — quasars: individual (OGLE050736.52-684751.7, OGLE050833.29-685427.5, OGLE050924.05-672124.1, OGLE051853.19-690217.7) X-rays: general

\section{Introduction}

There are a number of reasons that make quasars behind the Large Magellanic Cloud (LMC) - as well as other nearby galaxies - very interesting. Among other, they provide a good inertial reference system (e.g. Anguita, Loyola \& Pedreros 2000) to measure proper motion of the LMC. Also, such quasars can provide a line-of-sight probe of the interstellar medium in the LMC (e.g. Bowen, Blades \& Pettini 1995; Haberl et al. 2001; Kahabka, de Boer, \& Brün 2001). One aspect of such studies is to investigate the dust-to-gas ratio in galaxies (e.g. Fall \& Pei 1989).

There are only a dozen or so of publicly known quasars in the general direction of the LMC. All these quasars are located away from the bar of the LMC, in fairly sparse stellar fields. One was found by Blanco \& Heathcote (1986) via a grism survey. Crampton et al. (1997; see also Kahabka, de Boer, \& Brüns 2001) listed several other. In their analysis of the LMC proper motions, Anguita,

\footnotetext{
${ }^{1}$ Based on observations collected at the Magellan Baade 6.5-m telescope.

${ }^{2}$ Harvard-Smithsonian Center for Astrophysics, 60 Garden Street, Cambridge MA 02138, USA, e-mail: [adobrzycki,lmacri,kstanek]@cfa.harvard.edu

${ }^{3}$ Present address: Department of Astrophysics, University of Nijmegen, PO Box 9010, 6500 GL Nijmegen, The Netherlands, e-mail: pgroot@astro.kun.nl.
} 
Loyola, \& Pedreros (2000) show a sample of three quasars, including that of Blanco \& Heathcote (1986).

Drake et al. (2001) are currently pursuing a project of the study of the proper motions using quasars found in MACHO data. They quote a sample of $~ 30$ quasars in the dense stellar regions of the LMC, pre-selected using their variability as observed by the MACHO project, and then confirmed spectroscopically. However, the positions of those quasars are not publicly available.

A good starting point for searching for quasars is to utilize the fact that they are often bright in $\mathrm{X}$-rays, and a considerable number of quasars have been found in that way (including the ones from Crampton et al. 1997). However, the limited spatial resolution of earlier X-ray missions rendered this method impractical for the dense parts of the LMC, where a single X-ray source would have many optical candidates in its error box. The launch of the Chandra X-ray Observatory (Weisskopf et al. 2000) made application of this method to the LMC possible. Chandra's superb spatial resolution and excellent positional accuracy significantly reduce the source confusion problem.

Another characteristic that was used in quasar searches was their irregular variability. Several quasar surveys utilizing variability were performed or are on-going (e.g. Drake et al. 2001; Meusinger \& Brunzendorf 2001; Rengstorf et al. 2001).

Between 1997 and 2001, large parts of the Large Magellanic Cloud were monitored for microlensing events by the Optical Gravitational Lensing Experiment (OGLE-II: Udalski, Kubiak \& Szymański 1997). Udalski et al. (2000) released photometry and astrometry of more than seven million objects from the central parts of the $\mathrm{LMC}^{4}$. In addition, a large catalog of 68,000 variable objects observed by OGLE-II in both the LMC and the SMC was prepared by Żebruń et al. $(2001)^{5}$, based on a version of the image subtraction software (Alard \& Lupton 1998) developed by Woźniak (2000).

The availability of Chandra observations of fields in the LMC, combined with the availability of the OGLE database, allow combining the two methods in a search for quasars in the dense regions of the LMC. The Chandra detectors have a relatively large field of view and each pointing yields dozens of serendipitous sources.

\section{The archival data}

We searched the Chandra archive for imaging (i.e. with no grating) observations whose pointings would coincide with OGLE fields. At present, there are three such observations that are publicly available, ObsIDs 118, 125, and 776. A fourth observation fulfilling this criterion, ObsID 1991, was kindly provided to us by the P.I., K. Borkowski. All four observations were done

\footnotetext{
${ }^{4}$ Data available from ftp://bulge.princeton.edu/ogle/ogle2/maps/lmc/

${ }^{5}$ Data available from http://bulge.princeton.edu/ ogle/ogle2/dia/
} 
with ACIS as the focal plane detector, in various chip configurations. A single ACIS observation typically covers $0.12 \mathrm{deg}^{2}$. There was no overlap between the observations.

We reduced and analyzed the X-ray data using tools available in the CIAO 2.2.1 and SHERPA software packages ${ }^{6}$. We cleaned the electronic streaks in ACIS-S4 chip using destreak, and we searched the data for serendipitous point sources using the sliding cell tool celldetect; of the three detect tools available in CIAO this one is the most robust when it comes to the detection of point sources. We utilized the relation between the off-axis angle and signal-to-noise threshold from Dobrzycki et al. (2000). Overall, we identified 361 X-ray sources in our four Chandra observations. For each source, we then identified both the closest OGLE object and the closest OGLE variable. Some of the data available from the Chandra archive were processed before the best Chandra pointing calibration was available, and we used 5 arcsec as the threshold for the position match. We ended up with a list of 110 OGLE objects meeting those criteria and they formed a list of candidates for followup spectroscopy.

\section{Observations}

The optical spectra were obtained on 2002 January 22-23 with the Magellan Baade 6-5 meter telescope. We observed 35 objects out of 110 available candidates; the observed objects were primarily the brightest in X-rays.

We used the LDSS-2 imaging spectrograph. The instrument uses a $2048 \times 2048$ SITe\# 1 CCD with a scale of $0.38 \mathrm{arcsec} / \mathrm{pixel}$, a gain of $1 e^{-} / \mathrm{ADU}$, and a readout noise of $7 e^{-}$. The slit width was $1.03 \mathrm{arcsec}$ and the grism setting was $300 \mathrm{l} / \mathrm{mm}$, yielding a nominal resolution of $13.3 \AA$. Exposure times ranged from 300 to 600 seconds. All observations were carried out at the parallactic angle. Additionally, two spectrophotometric standards were observed: LTT 1788 and LTT 4816 (Hamuy et al. 1992). Following each observation, a He-Ne arc lamp spectrum was acquired for wavelength calibration purposes. Spectra were reduced in the standard way using IRAF.

\section{New quasars}

Out of 35 CXO/OGLE objects observed in January 2002, four turned out to be new quasars. In principle, this gives a $11 \%$ efficiency for the method for searching for quasars, but this value is, of course, subject to low number statistics. Table 1 contains a summary of the optical properties of the four quasars. We note that during the observation we could clearly identify the host galaxy for OGLE050924.05-672124.1, the one with lowest emission redshift $\left(z_{\mathrm{em}}=0.26\right)$.

We present the optical spectra of our quasars in Figure 1. In all of them, several emission

\footnotetext{
${ }^{6}$ http://cxc.harvard.edu/ciao/
} 
Table 1. Quasar optical data.

\begin{tabular}{llccc}
\hline \hline OGLE ID/Coords. $^{\mathrm{a}}$ & OGLE field & Redshift & $\begin{array}{c}V \\
{[\mathrm{mag}]}\end{array}$ & Remarks $^{\mathrm{b}}$ \\
\hline $050736.52-684751.7$ & SC11 & 0.53 & 19.81 & \\
$050833.29-685427.5$ & SC11 & 0.61 & 19.00 & $(1)$ \\
$050924.05-672124.1$ & SC25 & 0.26 & 20.27 & $(2)$ \\
$051853.19-690217.7$ & SC7 & 1.63 & 20.13 & \\
\hline
\end{tabular}

${ }^{a}$ OGLE ID contains J2000.0 equatorial coordinates.

b(1) Variable in Żebruń et al. 2001; (2) AGN host galaxy visible.

Table 2. Quasar X-ray data.

\begin{tabular}{ccccccccc}
\hline \hline Coords. $^{\mathrm{a}}$ & ObsID $^{\mathrm{b}}$ & $\operatorname{Exp}^{\mathrm{c}}$ & $N_{X}{ }^{\mathrm{d}}$ & $N_{\mathrm{H}, \mathrm{ATCA}}{ }^{\mathrm{e}}$ & $N_{\mathrm{H}, \mathrm{X}}{ }^{\mathrm{f}}$ & $\Gamma^{\mathrm{g}}$ & Norm $^{\mathrm{h}}$ & Remarks $^{\mathrm{i}}$ \\
\hline $050736.30-684751.9$ & 125 & 37.2 & 340 & 1.99 & $<6.0$ & $1.57 \pm 0.69$ & $1.7 \pm 1.0$ & $(1)$ \\
$050833.18-685427.9$ & 125 & 37.2 & 890 & 2.42 & $1.47 \pm 0.57$ & $2.19 \pm 0.24$ & $7.3 \pm 1.4$ & $(2)$ \\
$050923.94-672123.6$ & 776 & 49.6 & 440 & 2.27 & $1.93 \pm 0.93$ & $2.24 \pm 0.41$ & $2.8 \pm 0.9$ & $(3)$ \\
$051853.02-690217.6$ & 118 & 39.7 & 120 & 1.81 & $<8.3$ & $2.08 \pm 0.76$ & $0.6 \pm 0.5$ & \\
\hline
\end{tabular}

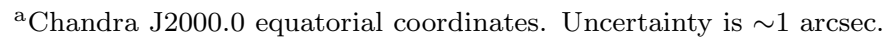

${ }^{\mathrm{b}}$ Chandra observation ID.

${ }^{\mathrm{c}}$ Chandra exposure time, in ks.

${ }^{\mathrm{d}}$ Net source events after background subtraction.

${ }^{\mathrm{e}} \mathrm{LMC}$ HI column density, in $10^{21} \mathrm{~cm}^{-2}$, from ATCA observations by Kim et al 1998. Values are \pm 0.01 .

${ }^{\mathrm{f}} \mathrm{LMC}$ absorbing column, in $10^{21} \mathrm{~cm}^{-2}$, from spectral fit. "<" indicates 3- $\sigma$ upper limit.

gPhoton spectral index, from spectral fit.

${ }^{\mathrm{h}}$ Power law normalization at $1 \mathrm{keV}$, in $10^{-5}$ photons $\mathrm{cm}^{-2} \mathrm{~s}^{-1} \mathrm{keV}^{-1}$, from spectral fit.

iHaberl \& Pietsch 1999 and Sasaki et al. 2000 info: (1) [HP99] 724, [SHP2000] 35, classified as "hard" (but not as "AGN"); (2) [HP99] 756, [SHP2000] 40; (3) [HP99] 523. 

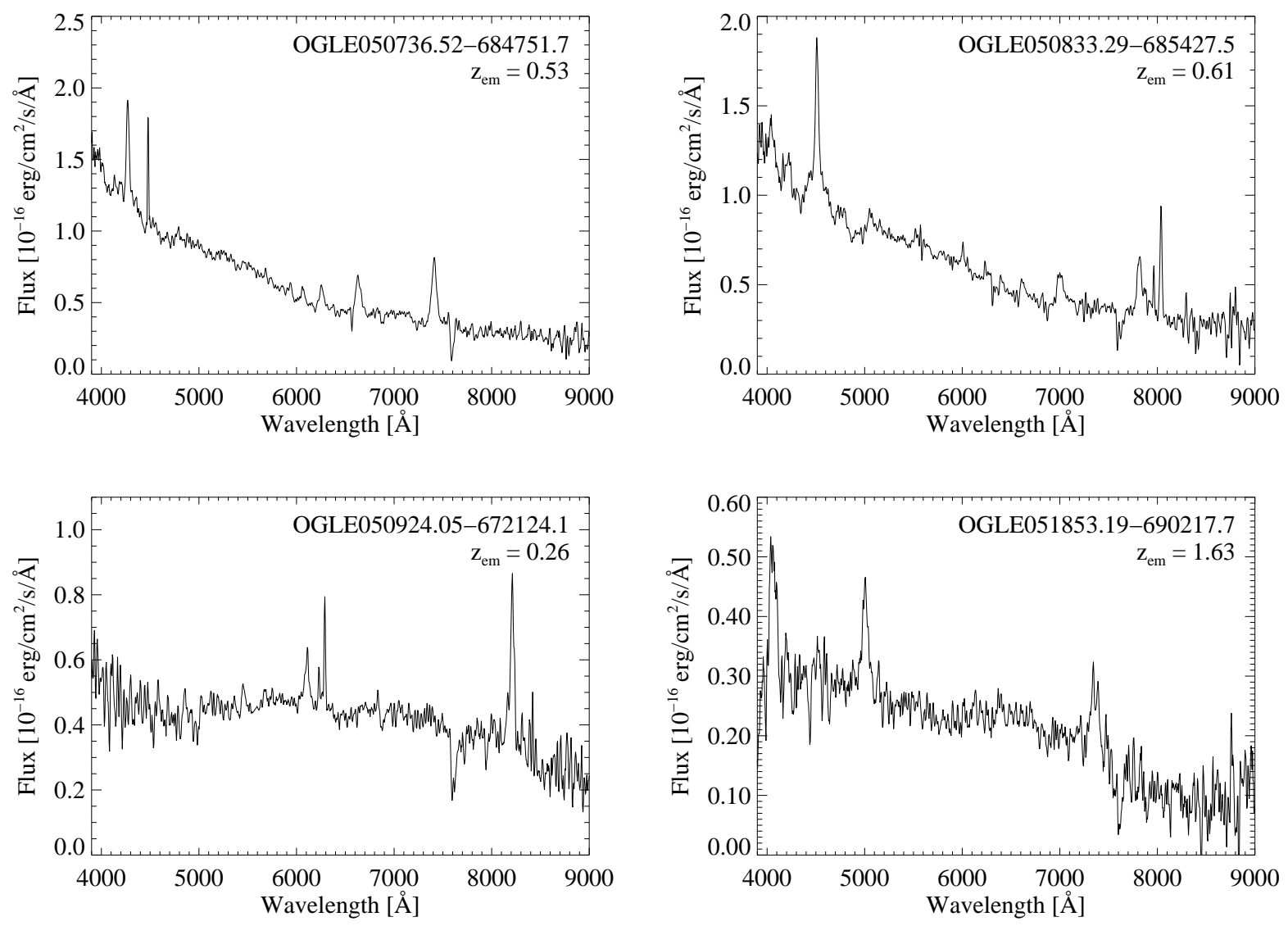

Fig. 1. - Optical spectra of the quasars, taken with the Magellan Baade 6.5-meter telescope. For presentation, the spectra were smoothed with a 3-pixel box.

lines are clearly visible, allowing unambiguous determinations of redshifts. All spectra show typical blue continua. One somewhat unusual feature is the apparent lack of [O III] emission lines in OGLE050736.52-684751.7 (upper left panel in Fig. 1), but at $z_{\mathrm{em}}=0.53$ they may have been affected by the strong $\mathrm{O}_{2}$ atmospheric band, which is clearly visible in the spectra.

We note that only one of the quasars, OGLE050833.29-685427.5, has been identified by OGLE as a variable. Its light curve clearly shows variability with the amplitude of $\sim 0.4$ mag. Post factum, we examined the light curves (provided to us by A. Udalski) of the other objects. The quasars are relatively faint, and the light curves are rather noisy, although they qualitatively suggest that the objects may be variable.

Table 2 contains a summary of the X-ray properties of the objects. We analyzed the X-ray data using tools from CIAO 2.2 and SHERPA. We reprocessed the observations using Chandra calibration database (CALDB) ver. 2.11. That allowed us to get corrected X-ray positions of sources, and we found them to agree well with the optical positions. We note that the positions 
of three of the quasars coincide with unidentified X-ray sources in the lists of ROSAT LMC X-ray sources from Haberl \& Pietsch (1999) and Sasaki, Haberl, \& Pietsch (2000); see Table 2.

The net number of X-ray events collected from four quasars range between $\sim 100$ and $\sim 900$, which is sufficient to establish basic X-ray properties of the sources. In addition to that, one can take advantage of the location of the quasars and - at least for the strongest of our quasars attempt to estimate the absorbing column in the LMC.

We extracted source and background spectra from regions around the quasars using dmextract and then performed spectral fits with SHERPA. Pile-up in ACIS is not a problem here, both because the source count rates are low and because all sources are far off-axis. We excluded data with $E<0.4 \mathrm{keV}$, since there are large calibration uncertainties for soft X-rays.

We fitted each spectrum assuming the intrinsic quasar spectrum to be a power law. We assumed fixed Galactic absorption towards the LMC, $N_{H}=5.5 \times 10^{21} \mathrm{~cm}^{-2}$ (Schwering \& Israel 1991), and we allowed for additional absorption from the LMC. In all cases, this model gave adequate fits. The derived spectral X-ray properties (Tab. 2) are within typical ranges for quasars (e.g. Fiore et al. 1998; Reeves \& Turner 2000).

We got a meaningful measurement of LMC absorption only for the two strongest X-ray sources among our four quasars. For the two weaker sources, the uncertainty in the LMC absorbing column was larger than the derived value; for those objects we list the $3 \sigma$ upper limits in Table 2. For comparison, in Table 2 we list the hydrogen column density from the Australia Telescope Compact Array (ATCA) $21 \mathrm{~cm}$ observations by Kim et al. (1998). The values derived from the X-ray spectral fits are in reasonable agreement with the measurements of the hydrogen content in the LMC. Our value for OGLE050833.29-685427.5 differs from the ATCA result by $\sim 2 \sigma$, but there are several factors that can readily explain the difference. First, the assumed value for the Galactic absorption may be overestimated, resulting in underestimating the LMC component of the absorbing column. Second, it is well established (e.g. Kim et al. 1998) that the distribution of hydrogen in the LMC is far from homogeneous. The measurements from Kim et al. (1998) are effectively averaged over spatial resolution element of $\sim 1$ arcmin (15 pc at the LMC), while the X-ray absorption is probing a specific line of sight.

\section{Summary and discussion}

We identified four previously unknown quasars behind the Large Magellanic Cloud, including one quasar at relatively high redshift, $z_{\mathrm{em}}=1.63$, by combining publicly available Chandra and OGLE data and ground-based followup spectroscopy. While the underlying idea in this project was to search for quasars based on both their X-ray positions and variability, we note that only one of our four quasars was positively identified by OGLE as a variable object. The validity of our assumption that the combined Chandra/OGLE pointings are good enough to accurately pinpoint quasar positions is an excellent starting point for future studies of X-ray selected quasars behind 
the LMC. So far, we have found four quasars in $\sim 0.5 \mathrm{deg}^{2}$ covered by the four analyzed Chandra observations, which compares favorably with $\sim 30$ quasars in $\sim 11 \mathrm{deg}^{2}$, discovered by the MACHO project on the basis of their variability

The intrinsic X-ray and optical properties of all four quasars are quite typical. All four quasars are relatively faint $(V=19-20)$, which, at least at present, makes them unlikely targets for highresolution observations, especially with the Hubble Space Telescope. HST observations would be necessary for the analysis of the most important absorption lines, such as Ly- $\alpha, \mathrm{C}$ IV or $\mathrm{Mg}$ II, which for LMC occur in the UV part of the spectrum, below the atmospheric break. However, the quasars will be very attractive targets for the new generation of large telescopes, as well as the NGST.

Three of the quasars are well positioned to become reference points for proper motion studies. They are in dense fields: OGLE050736.52-684751.7 and OGLE050833.29-685427.5 are near the edge of the LMC bar and OGLE051853.19-690217.7 is near the center of the bar. The fourth quasar, OGLE050924.05-672124.1, is outside of the LMC bar, and it is also the one for which the host galaxy is visible, limiting somewhat its application for this type of project.

We add that the followup spectroscopy of the CXO/OGLE candidates revealed several other interesting objects, such as very hot stars, X-ray binaries, etc. We will present the analysis of those objects in a forthcoming paper.

We would like to thank B. Paczyński, A. Siemiginowska and the referee for helpful comments. We would also like to thank K. Borkowski for allowing us to use his Chandra observation prior to it becoming public, S. Kim and A. Udalski for providing us with unpublished data, and T. Anselowitz for help with processing the X-ray observations. We are grateful to the OGLE collaboration for putting their large data sets in public domain. This research has made use of the NASA/IPAC Extragalactic Database (NED) which is operated by the Jet Propulsion Laboratory, California Institute of Technology, under contract with NASA, and of the SIMBAD database, operated at CDS, Strasbourg, France. AD acknowledges support from NASA Contract No. NAS8-39073 (CXC). PJG was supported by a CfA fellowship.

\section{REFERENCES}

Alard, C., \& Lupton, R. H. 1998, ApJ, 503, 325

Anguita, C., Loyola, P.,\& Pedreros, M. H. 2000, AJ, 120, 845

Blanco, V. M., \& Heathcote, S. 1986, PASP, 98, 635

Bowen, D. V., Blades, J. C., \& Pettini, M. 1995, ApJ, 448, 634

Crampton, D., Gussie, G., Cowley, A. P., \& Schmidtke, P. C. 1997, AJ, 114, 2353

Dobrzycki, A., Jessop, H., Calderwood, T. J., \& Harris, D. E. 2000, AAS/HEAD, 32, 2708 
Drake, A. J., et al. (MACHO Collaboration) 2001, AAS Meeting, 199, 52.05

Fall, S. M., \& Pei, Y. C. 1989, ApJ, 337, 7

Fiore, F., Elvis, M., Giommi, P., \& Padovani, P. 1998, ApJ, 492, 79

Haberl, F., Dennerl, K., Filipović, M. D., Aschenbach, B., Pietsch, W., \& Trümper, J. 2001, A\&A, 365, L208

Haberl, F. \& Pietsch W. 1999, A\&AS, 139, 277

Hamuy, M., Walker, A. R., Suntzeff, N. B., Gigoux, P., Heathcote, S. R., \& Phillips, M. M. 1992, PASP, 104, 533

Kahabka, P., de Boer, K. S., \& Brüns, C. 2001, A\&A, 371, 816

Kim, S., Staveley-Smith, L., Dopita, M. A., Freeman, K. C., Sault, R. J., Kesteven, M. J., \& McConnell, D. 1998, ApJ, 503, 674

Meusinger, H. \& Brunzendorf, J. 2001, A\&A, 374, 878

Reeves, J. N., \& Turner, M. J. L. 2000, MNRAS, 316, 234

Rengstorf, A. W., et al. (QUEST Collaboration) 2001, AAS Meeting, 199, 138.07

Sasaki, M., Haberl, F., \& Pietsch, W. 2000, A\&AS, 143, 391

Schwering, P. B. W., \& Israel, F. P. 1991, A\&A, 246, 231

Udalski, A., Kubiak, M., \& Szymański, M. 1997, AcA, 47, 319

Udalski, A., Szymański, M., Kubiak, M., Pietrzyński, G., Soszyński, I., Woźniak, P., \& Żebruń, K. 2000, AcA, 50, 307

Weisskopf, M. C., et al. 2000, Proc. SPIE, 4012, 2

Woźniak, P. R. 2000, AcA, 50, 421

Żebruń, K., et al. (OGLE Collaboration) 2001, AcA, 51, 317 